\title{
Remembrances of Walter Craig
}

\section{Walter Craig (1953-2019), a Passionate Mathematician and a Dear Friend}

\section{Catherine Sulem and C. Eugene Wayne}

Walter Craig, one of the leading analysts and applied mathematicians of his generation, died on January 18, 2019, in Hamilton, Ontario. His parents, William Craig and Julia Rebecca Dwight Wilson, lived in State College, Pennsylvania, where he was born on November 28, 1953. His father was at the time a professor in the mathematics department at Penn State. The family moved to Berkeley in 1959, where his father became a professor at the University of California, Berkeley, and it was in Berkeley that Walter grew up, along with his parents and three sisters, in a house not far from the Berkeley campus. Walter attended public schools through the turbulent years of the 1960s, and upon graduation in 1971, he spent a year at the University of California, Santa Cruz, before moving back to continue his undergraduate degree at Berkeley. After only a year as a student at Berkeley, however, Walter decided to pursue his love of music and spent two years working as a professional jazz bass player in the Bay Area. Walter's affection for music continued throughout his life, and his later years saw him transposing Bach's cello suites to play on his bass or guitar.

Returning to college, Walter finished his bachelor's degree in mathematics at Berkeley in 1977, after which he moved to New York to attend graduate school at the

Catherine Sulem is a professor of mathematics at the University of Toronto. Her email address is sulemamath. utoronto.ca.

C. Eugene Wayne is a professor of mathematics at Boston University. His email address is cew@bu.edu.

Communicated by Notices Associate Editor Reza Malek-Madani.

For permission to reprint this article, please contact:

reprint-permission@ams.org.

DOI: https://doi.org/10.1090/noti2071

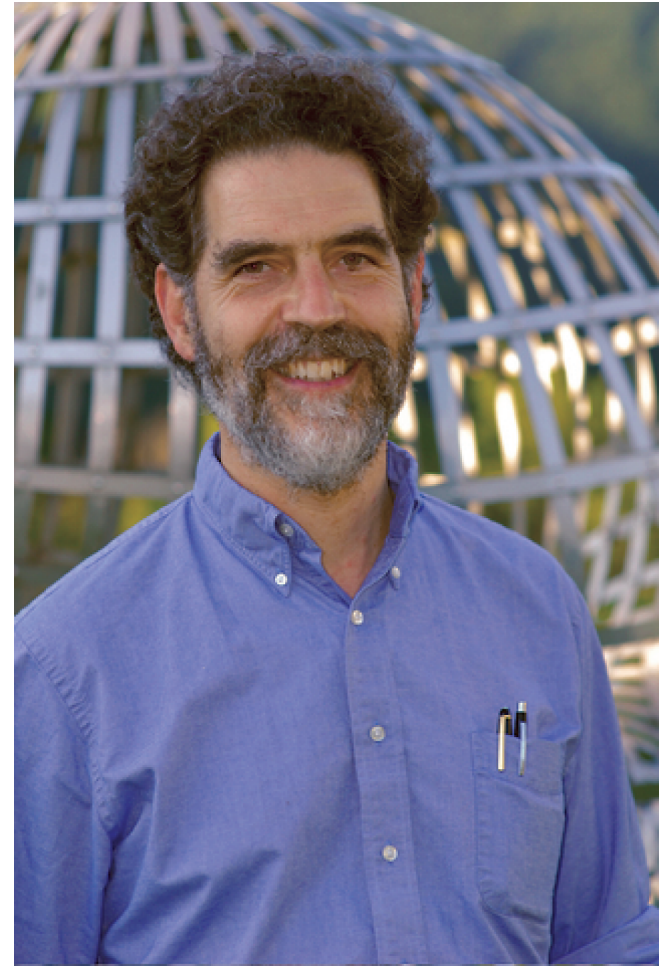

Figure 1. Walter at an Oberwolfach meeting in 2007.

Courant Institute. Walter still found time to play occasionally in the jazz clubs in Greenwich Village, but he wasted no time in finishing his PhD "A Bifurcation Theory for Periodic Dissipative Wave Equations" under the direction of Louis Nirenberg.

After graduate school, Walter returned to the West Coast, where he was first a Bantrell Instructor at Cal Tech for three years and then an assistant professor at Stanford. While at Cal Tech, he published several papers on the spectral properties of Schrödinger operators. Throughout his life, Walter had a strong sense of intellectual independence. It was during this period that he began to work on the water wave problem, an area that was not particularly fashionable at that time. His first published paper on this problem, a 200-page pioneering work, transformed the field of freesurface waves, and he pursued this topic throughout his 
career. In addition to being both mathematically and musically inclined, Walter was also mechanically gifted, and people who knew him during his time in California often tell stories of the motorcycle and old English sports car that he repaired and drove enthusiastically around Palo Alto. It was in Stanford that Walter met Deirdre Haskell, who was then a mathematics graduate student at Stanford and whom he later married.

In 1988, Walter crossed the country once more to become associate professor at Brown, being promoted to professor in 1991 and serving as department chair from 1997 to 2000. During this period, Walter's work also began to be recognized with a series of national and international awards that continued throughout his career. From 1988 to 1993 he was an NSF Presidential Young Investigator, and he also received a Sloan Fellowship in 1988.

In 2000, Walter, Deirdre, and their daughter Zoé moved to Canada. Walter was offered a Tier 1 Canada Research Chair in Mathematical Analysis and its Applications, the very first appointment through the CRC program in the country, and Deirdre was offered a professorship, both in the mathematics department of McMaster University in Hamilton. He spent the rest of his career in Hamilton, with the exception of two years when he served as director of the Fields Institute. He was named Fellow of the Royal Society of Canada in 2007, Fellow of the American Association for the Advancement of Science in 2008, and was awarded the prestigious Killam Research Fellowship in 2009 from the Canada Council for the Arts. He was one of the inaugural group of Fellows of the American Mathematical Society named in 2013. His contributions at McMaster University were recognized with conferral of the title Distinguished University Professor in 2016.

During his time in Canada, Walter organized three yearlong thematic programs at the Fields Institute and was the driving force behind innumerable conferences and workshops around the world. Between 2001 and 2005, he organized an annual PDE conference for junior mathematicians taking place in Ontario. Indeed, Walter was an exceptional teacher and mentor who educated and supervised many undergraduate and graduate students, postdoctoral fellows, and young mathematicians. Many of them have gone on to become accomplished scholars in various countries around the world. Recalling her years as Walter's postdoctoral fellow, Livia Corsi, now assistant professor at University of Roma Tre, says: "I will always be grateful for having the opportunity to be Walter's postdoc for two years. He was simply the best mentor I could hope for, both mathematically and personally. We were working on a very hard problem and I was getting a little frustrated. In those moments, he would tell me the story of a colleague of his father, sitting on a bench, looking sad and

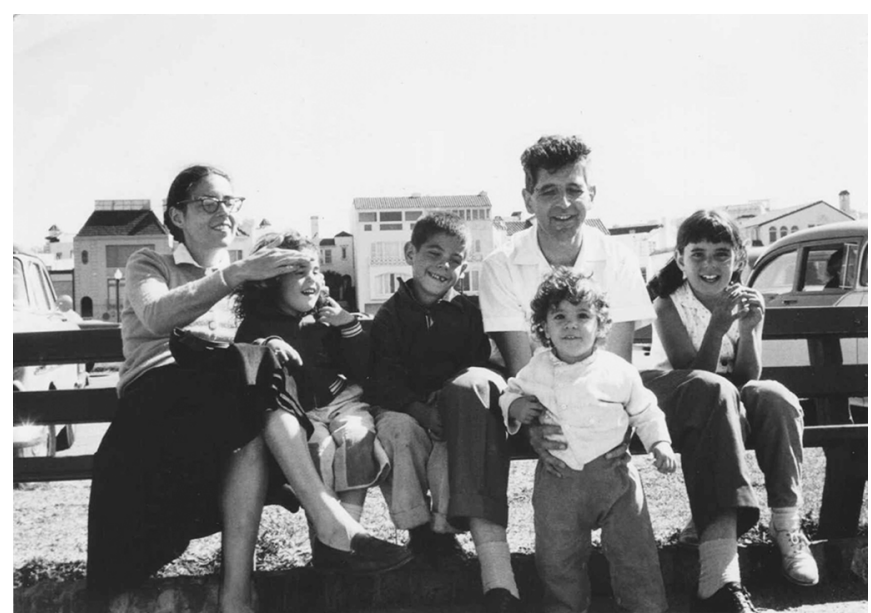

Figure 2. Walter with his parents and sisters in the Bay Area (California) in 1961.

saying, 'I don't have problems!', and remind me that having problems is precisely what makes us (mathematicians) feel happy and alive."

Walter was a mathematical analyst with eclectic interests who enjoyed working with colleagues from different areas of expertise. His research topics included nonlinear PDEs, infinite-dimensional Hamiltonian systems, Schrödinger operators and spectral theory, water waves and its applications, general relativity, and cosmology. He had an extensive scientific culture and was skillful at communicating with people from different scientific backgrounds. It was common to see him at the center of lively conversations in English or French during seminars, conferences, or workshops. Walter was passionate about science and mathematics. He had an independent mind and a sharp intellect. He was also warm and caring. He loved travelling and meeting mathematicians all over the world and was a constant source of inspiration for people around him.

Despite the cancer diagnosis in the fall of 2014 and the following rigorous treatments and doctor's appointments, Walter continued to live his life fully with optimism, courage, and generosity. In December 2018, he had just completed his textbook A Course on Partial Differential Equations for the AMS series Graduate Studies in Mathematics when the illness took a bad turn. In this book, which covers basic notions of PDEs and is aimed at beginning graduate students, Walter presents his personal point of view of the topics, elegantly combining theory, phenomenology, and applications. Walter's artistic inclination and sense of humour are also apparent, for example, in the analysis of musical instruments in the chapter on wave equations and the special pictures at the beginning of each chapter. The picture for the chapter on dispersion is Mystic, Walter's beloved cat! The book appeared the week following his death. 
A celebration of Walter's life was held in Hamilton on March 16, 2019, and was attended by more than one hundred friends and family members. A volume of the new journal Water Waves dedicated to Walter will appear in 2020. Other events, including the 12th Americas Conference on Differential Equations and Nonlinear Analysis (Americas XII) in December 2019 at Guanajuato and a workshop on "surface wave hydrodynamics" at the Fields Institute in fall 2020 will take place to honor his mathematical legacy. We will always keep loving memories of Walter in our hearts.

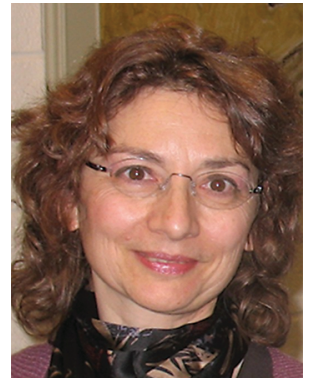

Catherine Sulem

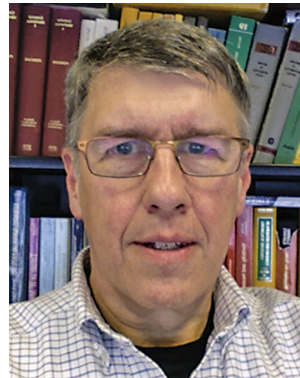

C. Eugene Wayne

\section{Walter's Pioneering Work on Water Waves}

\section{Philippe Guyenne and Catherine Sulem}

Walter was fascinated by ocean waves and often started his lectures with quotes from Homer's Odyssey or James Joyce's Ulysses. Throughout his career, he made significant contributions to the rigorous analysis, mathematical modeling, and numerical simulation of various aspects of the water wave problem. The classical theory for this problem involves elements of two mathematical topics that were dear to Walter: partial differential equations (PDEs) and Hamiltonian dynamical systems. His first contribution in this area was a single-authored 200-page paper [6], published soon after he joined Stanford University as an assistant professor, which provides a rigorous justification of the Korteweg-de Vries (KdV) approximation. This was a groundbreaking achievement, especially back in the 1980 s when there were very few rigorous results, and it remains a major reference in the literature on the mathematical theory of water waves. It is in this paper that Walter first introduced the so-called Dirichlet-Neumann operator (DNO) to reformulate the governing equations in

Philippe Guyenne is a professor of mathematics at the University of Delaware. His email address is guyenne@ude1. edu. a lower-dimensional form that is more suitable for analysis. He was a strong advocate of this powerful mathematical tool and used it extensively in his subsequent work on the water wave problem. In a detailed MathSciNet review (MR1780702) of two papers, one by G. Schneider and G. Wayne and the other by D. Christodoulou and H. Lindblad, Walter presents his point of view and a brief history of rigorous results on the initial value problem for water waves starting with the fundamental 1974 paper by Nalimov for initial data in Sobolev spaces.

Hamiltonian formulation and Dirichlet-Neumann operator. Tackling the water wave problem entails considerable challenges, both mathematically and numerically, because of its multidimensionality and nonlinearity. More notably, it is a free-boundary value problem in the sense that the free surface is to be found as part of the solution and the velocity field $\mathbf{u}(x, y, t)$ depends nonlocally on the moving boundary of the fluid domain. Consider an $(n+1)$ dimensional fluid ( $n=1$ or 2 ) bounded below by a flat rigid bottom $\{y=-h\}$ and above by a free surface defined by the graph $\Gamma(\eta)=\left\{y=\eta(x, t), x \in \mathbb{R}^{n}, t \geq 0\right\}$. Assume the fluid is incompressible, inviscid, and the flow is irrotational so that $\mathbf{u}=\nabla \varphi$, where the scalar function $\varphi(x, y, t)$ denotes the velocity potential. Under these conditions, $\mathrm{V}$. Zakharov [22] proved the remarkable property that the governing equations can be cast as a canonical Hamiltonian system

$$
\partial_{t} v=J \nabla_{v} \mathcal{H}(v), \quad v=\left(\begin{array}{c}
\eta \\
\xi
\end{array}\right), \quad J=\left(\begin{array}{rr}
0 & 1 \\
-1 & 0
\end{array}\right),
$$

where $\xi(x, t)=\varphi(x, \eta(x, t), t)$ is the trace of the velocity potential at the free surface, $J$ is a skew-symmetric matrix representing the symplectic structure of the system, and $\mathcal{H}$ is the Hamiltonian representing the total energy (which is conserved over time).

In [14], Walter together with C. Sulem made the key observation that the dependence on $\eta$ can be made more explicit by introducing the DNO, $G(\eta): \xi \mapsto\left(-\nabla_{x} \eta, 1\right)^{\top}$. $\left.\nabla \varphi\right|_{y=\eta}$ from $H^{1}(\Gamma)$ to $L^{2}(\Gamma)$, which is the singular integral operator that takes Dirichlet data $\xi$, solves Laplace's equation for $\varphi$ inside the fluid domain, and returns the normal fluid velocity at the free surface. With this in hand, the Hamiltonian can be written as

$$
\mathcal{H}(\eta, \xi)=\frac{1}{2} \int_{\mathbb{R}^{n}}\left[\xi G(\eta) \xi+g \eta^{2}\right] d x:=\mathcal{K}+\mathcal{P},
$$

where $\mathcal{K}$ and $\mathcal{P}$ denote the kinetic and potential energies, respectively, and equation (1) takes a closed form in terms of surface variables alone. The only restoring force considered here is gravity $g$ (surface tension is neglected). This formulation of the water wave equations has a lot of appeal due to its reduced dimensionality and has been adopted 
in a countless number of studies, including recent notable results on well-posedness and rigorous justification of asymptotic models $[18,19]$. The canonical variables can be obtained from classical mechanics principles via a straightforward calculation using the DNO and the Lagrangian $\mathcal{L}:=\mathcal{K}-\mathcal{P}$ for water waves in terms of tangent-space variables $(\eta, \dot{\eta})$ where $\dot{\eta}=G(\eta) \xi$ by virtue of the free-surface kinematic condition. The canonical conjugate variables are then deduced from $\left(\eta, \partial_{\dot{\eta}} \mathcal{L}\right)=\left(\eta, G^{-1}(\eta) \dot{\eta}\right)=(\eta, \xi)$, which are precisely those introduced by Zakharov.

The DNO has several interesting properties. In particular, it is self-adjoint and depends analytically upon $\eta \in B_{R}(0) \subseteq C^{1}(\mathbb{R})$, where $B_{R}(0)$ is a ball of radius $R$ centered at the origin. Exploiting this fact, Walter and Catherine devised a perturbative procedure to evaluate the DNO via a Taylor series expansion

$$
G(\eta)=\sum_{j=0}^{\infty} G_{j}(\eta),
$$

where each Taylor term $G_{j}$ is homogeneous of degree $j$ in $\eta$ and can be determined recursively. For example, the first two terms are given by $G_{0}=D \tanh (h D)$ and $G_{1}=$ $D \cdot \eta D-G_{0} \eta G_{0}$, where $D=-i \nabla_{x}$ is a Fourier multiplier with Fourier symbol $k$. This series form of the DNO turns out to be well suited for both perturbation calculations and numerical simulations. These two aspects, combined with the fact that the DNO blends well into the Hamiltonian formulation (1) and can be placed on rigorous mathematical grounds, constitute a unique feature of the approach developed in [14]. This has helped produce a number of remarkable results in the past decade or so, some of which are presented in the next sections.

Direct numerical simulations. Noting that each term in (3) is a sum of concatenations of Fourier multipliers with powers of $\eta$, a pseudo-spectral scheme can be used to efficiently and accurately compute the DNO expansion via the fast Fourier transform. The computational cost can be estimated to be $O\left(M^{2} N \log N\right)$ per time step, where $N$ is the total number of grid points in $\mathbb{R}^{n}$ and $M \ll N$ is the truncation order of (3). The DNO analyticity guarantees exponential convergence of (3) for a sufficiently regular free surface, which implies that a small number of terms (typically $M<10$ ) is sufficient to achieve highly accurate results. Unlike boundary integral methods for solving Laplace problems, this numerical approach does not require assembling and solving any dense matrix system at every time step. Moreover, its implementation is insensitive to the spatial dimension $n$ and water depth $h$. For these reasons, it has played a key role in the advancement and popularity of so-called high-order spectral methods for the direct simulation of nonlinear dispersive
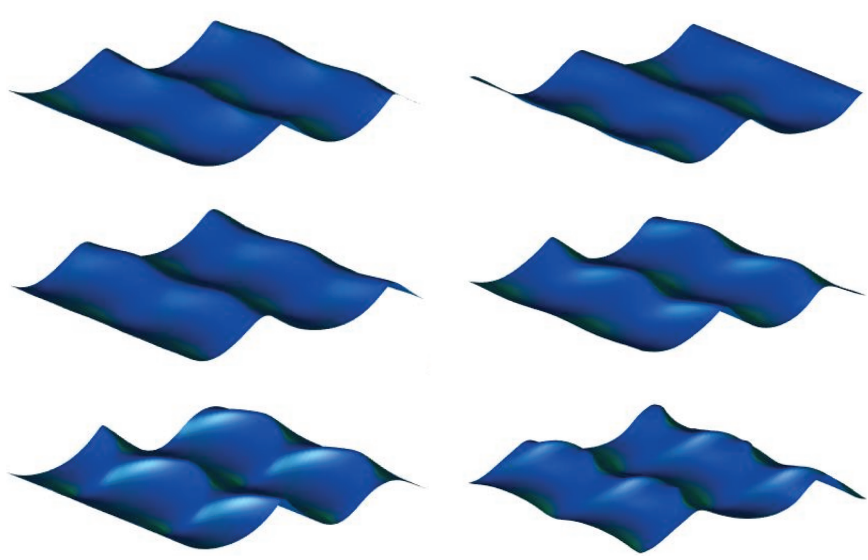

Figure 3. The development of 3D crescent waves from an initially perturbed Stokes wave.

water waves, which are now widely used by engineers and oceanographers.

Figures 3 and 4 present simulations based on this numerical method. Figure 3 shows the development of 3D crescent waves from an initially perturbed Stokes wave on deep water [21]. Figure 4 shows the head-on collision of two solitary waves of slightly different amplitudes on shallow water. The computations are compared with experiments conducted in the Pritchard Fluid Mechanics Laboratory at Penn State University. Walter was delighted by this collaborative work with J. Hammack and D. Henderson. The resulting paper [8] provides one of the most detailed studies of solitary wave interactions, combining mathematical, numerical, and experimental results.

Hamiltonian transformation theory and asymptotic models. The development and analysis of asymptotic models were of special interest to Walter, going back to [6]. The water wave problem is notorious for its wealth of reduced models that can be derived in various asymptotic limits. Walter was particularly supportive of the idea that such models should retain important structural properties of the original system, including energy conservation. It is thus desirable that they retain a Hamiltonian structure. Except for a number of canonical examples such as the nonlinear Schrödinger and Korteweg-de Vries equations, many existing models (especially at high truncation order or in complex physical settings) are not known to be Hamiltonian PDEs. This led Walter, with various collaborators over the years (e.g., M. Groves, H. Kalisch, and the present authors), to initiate a research program aimed at developing a systematic perturbation approach to derive Hamiltonian asymptotic models for water waves. This point of view would also provide a unified theoretical framework for further analysis of these models and a suitable starting point for possible inclusion of additional effects. While perturbation (and more generally 


\section{MEMORIAL TRIBUTE}
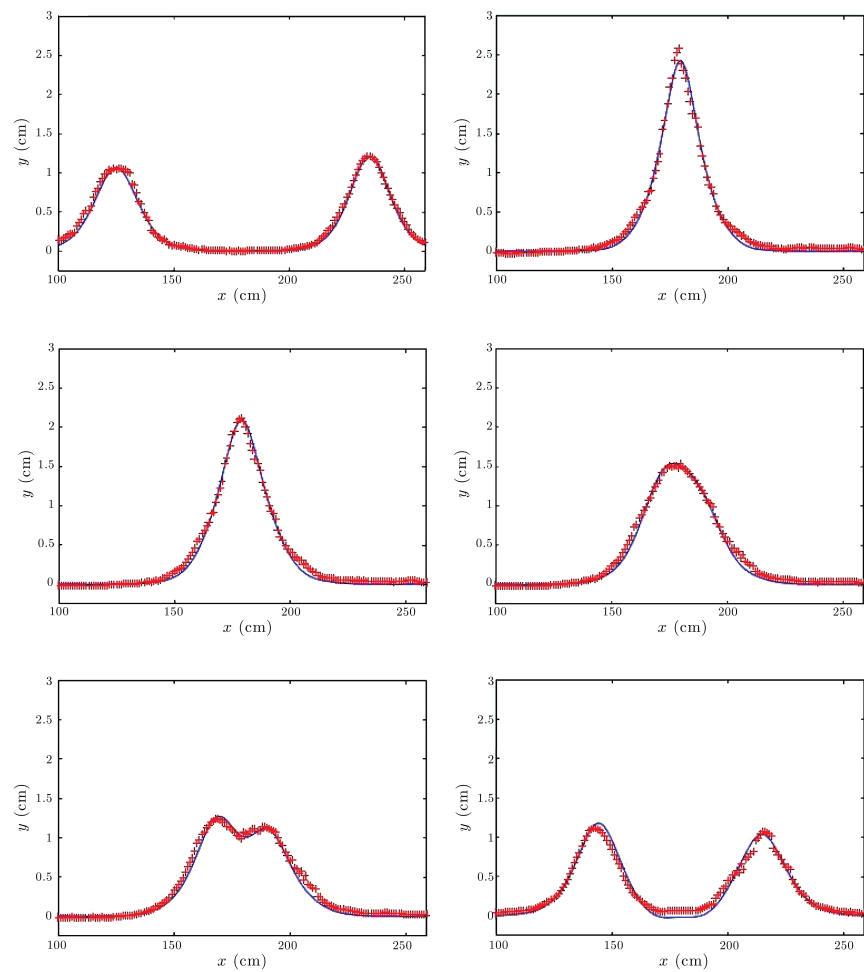

Figure 4. The head-on collision of two solitary waves. Experimental results (red crosses), numerical results (blue line).

transformation) theory is a basic well-established tool for finite-dimensional Hamiltonian systems, it is less understood in the context of PDEs and infinite-dimensional Hamiltonian systems.

There are two essential steps in this procedure: expansion of the Hamiltonian and transformation of the symplectic structure. Given a small parameter $\varepsilon$, it is natural to expand $\mathcal{H}$ in powers of $\varepsilon$ via (3) and approximate orbits $v(t ; \varepsilon)$ of (1) by those of the truncated problem $\partial_{t} v^{(m)}=J \nabla_{v}\left(\mathcal{H}^{(0)}+\varepsilon \mathcal{H}^{(1)}+\cdots+\varepsilon^{m} \mathcal{H}^{(m)}\right)$. For at least finite intervals of time, solutions $v^{(m)}(t ; \varepsilon)$ are expected to be $\varepsilon^{m}$ close to solutions of the full problem. Additionally, the changes of variables associated with a particular asymptotic regime modify the symplectic structure of the original system. Consider two phase spaces $V_{1}$ and $V_{2}$, with a symplectic structure on $V_{1}$ given by $J_{1}$. Let $\mathcal{H}_{1}: V_{1} \rightarrow \mathbb{R}$ be a Hamiltonian. A transformation $f: v_{1} \mapsto v_{2}$ from $V_{1}$ to $V_{2}$ gives rise to a Hamiltonian $\mathcal{H}_{2}\left(v_{2}\right)=\mathcal{H}_{2}\left(f\left(v_{1}\right)\right)=\mathcal{H}_{1}\left(v_{1}\right)$ defined on $V_{2}$. The corresponding vector field is transformed as

$$
\begin{aligned}
\partial_{t} v_{2} & =\left(\partial_{v_{1}} f\right)\left(\partial_{t} v_{1}\right)=\left(\partial_{v_{1}} f\right) J_{1} \nabla_{v_{1}} \mathcal{H}_{1} \\
& =\left(\partial_{v_{1}} f\right) J_{1}\left(\partial_{v_{1}} f\right)^{\top} \nabla_{v_{2}} \mathcal{H}_{2},
\end{aligned}
$$

where $J_{2}=\left(\partial_{v_{1}} f\right) J_{1}\left(\partial_{v_{1}} f\right)^{\top}$ is a symplectic structure defined on $V_{2}$.
For example, in dimension $n=1$, typical transformations associated with the long-wave (or shallow-water) regime include amplitude scaling $(\eta, \xi) \mapsto(\tilde{\eta}, \tilde{\xi})=$ $\left(\eta / \varepsilon^{2}, \xi / \varepsilon\right)$, where $J_{2}=\varepsilon^{-3} J_{1}$; spatial scaling $x \mapsto X=\varepsilon x$, where $J_{2}=\varepsilon J_{1}$; and change to elevation-velocity coordinates $(\eta, \xi) \mapsto(\eta, u)=\left(\eta, \partial_{X} \xi\right)$, where $J_{2}=\left(\begin{array}{cc}0 & -\partial_{X} \\ -\partial_{X} & 0\end{array}\right)$, which lead to Boussinesq-type equations for $\eta$ and $u$. Further transformations to accommodate a moving reference frame, together with solutions traveling in a preferred direction, ultimately yield the $\mathrm{KdV}$ equation and possibly higher-order versions of it.

In recent years, Walter paid increasing attention to problems that extend beyond the classical setting of wave propagation in a homogeneous medium. These include surface waves propagating over a variable bottom topography and surface waves interacting with internal waves in stratified flows. This was another trait of Walter's: in parallel to his mathematical analysis interests, he was aware of questions relevant to engineers and oceanographers. To add to the challenge, cases under consideration involve wave-bottom and wave-wave interactions across a wide range of length scales.

In $[10,20]$, an extension of the aforementioned Hamiltonian formulation to water waves on variable depth was proposed and investigated. Similarly, the bottom variations are encoded in the series form (3) of the DNO. The asymptotic regime of long waves propagating over bathymetry with shorter-scale variations was treated via homogenization of the Hamiltonian (2). For periodically varying topographies $(n=1)$, their effects were found to fully homogenize by virtue of the scale-separation lemma

$$
\int_{\mathbb{R}} p(X / \varepsilon) q(X) d X=\langle p\rangle \int_{\mathbb{R}} q(X) d X+O\left(\varepsilon^{\gamma}\right) \quad \forall \gamma>0,
$$

where $p(x)$ is any continuous periodic function with average value $\langle p\rangle$ and $q(X)$ is any Schwartz-class function. This led to a KdV equation with adjusted effective coefficients. On the other hand, for randomly varying topographies, their effects are stronger by virtue of the following lemma (in the sense of convergence in law):

$$
\begin{aligned}
\int_{\mathbb{R}} p & (X / \varepsilon, \omega) q(X) d X \\
& =\int_{\mathbb{R}}\left[\langle p\rangle+\sqrt{\varepsilon} \sigma_{p} \partial_{X} \mathcal{B}_{\omega}(X)\right] q(X) d X+o(\sqrt{\varepsilon}),
\end{aligned}
$$

where $p(x, \omega)$ is any stationary mixing ergodic process with expected value $\langle p\rangle$ and variance $\sigma_{p}^{2}$, and $\mathcal{B}_{\omega}(X)$ is Brownian motion. As a result, the problem does not fully homogenize and retains realization-dependent contributions, even to leading order, which affect both the speed and amplitude of the waves. As an application, these random effects may quantify the degree of uncertainty in our 
knowledge of the oceans' bathymetry and may explain systematic errors made in the forecasting of travel time of tsunamis.

In [9], Walter and collaborators developed a similar formalism for the Hamiltonian equations governing twolayer flows, where two DNOs are required, one for each layer. In particular, the DNO for the upper layer is defined as a matrix operator that encodes the coupling between the top free surface and the interface separating the two layers. This approach was used in [11] to describe the resonant interaction between internal waves and surface waves in a stratified ocean, in a scaling regime chosen to model physical phenomena such as the formation of rips and the mill-pond effect, which have been captured in in situ observations and on NASA photographs from the space shuttle. The presence of multiple length scales was again handled via homogenization, yielding a reduced system where the internal wave $r$ is modeled by a KdV equation $\partial_{\tau} r+a_{1} r \partial_{X} r+a_{2} \partial_{X}^{3} r=0$, with $\tau=\varepsilon \tau_{1}=\varepsilon^{3} t$, and the complex envelope $v\left(X, \tau_{1}\right)$ of the surface waves satisfies a linear Schrödinger equation $-i \partial_{\tau_{1}} v=-\delta^{2} \partial_{X}^{2} v+a_{3} r\left(X, \varepsilon \tau_{1}\right) v$, with an external potential given by the internal wave profile. All coefficients in these PDEs can be expressed in terms of two free parameters, the relative density $\rho_{1} / \rho$ and relative depth $h_{1} / h$ of the two layers. Under typical oceanic conditions, this Schrödinger equation is found to be in a semiclassical regime, and the surface signature of internal waves is generated by a process analogous to radiative absorption, providing a new analysis for the surface rips in terms of localized bound states. These two studies are perfect examples illustrating Walter's talent at developing elegant mathematical methods to tackle challenging physical problems and obtain results of both mathematical and practical interest with surprising connections to other scientific areas.

Walter was full of ideas and projects when, sadly, his life was cut short. Both authors of this article were fortunate to have enjoyed a long friendship and continued collaboration with Walter over many years. He was a source of inspiration and a role model for young mathematicians, including the many students and postdocs he supervised. He was generous with his time, support, and ideas, and his enthusiasm when tackling mathematical problems was contagious. He will leave a lasting legacy in the scientific community.

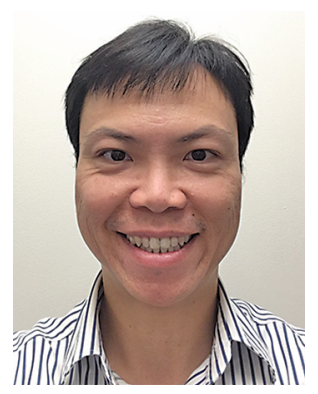

Philippe Guyenne

\section{Small Divisors, Resonance, and Their Consequences in Partial Differential Equations}

\section{Eugene Wayne}

Most of Walter Craig's mathematical research focussed on nonlinear partial differential equations (PDEs). He felt that as questions of existence and uniqueness of such equations were increasingly well understood, one should ask more refined questions about the existence and properties of special families of solutions such as solitary waves or periodic solutions. Indeed, his $\mathrm{PhD}$ thesis focussed on the existence of temporally periodic solutions of damped hyperbolic equations, which he treated with the aid of ideas from bifurcation theory. The use of ideas from finitedimensional dynamical systems theory, modified to suit an infinite-dimensional context, was something that recurred frequently throughout Walter's work.

If instead of dissipative PDEs of the type Walter considered in his thesis, one considers undamped, Hamiltonian PDEs, the search for periodic solutions is often frustrated by the presence of "small divisors." The development of methods to treat small divisors in infinite dimensions was a focus of Walter's research for a number of years, and he made very important contributions to understanding the effects of these small divisors and the resonances that underlie them in a variety of different contexts.

As a simple example, consider the nonlinear wave equation with periodic boundary conditions:

$$
u_{t t}=u_{x x}-\epsilon u^{3}, \quad u(x+2 \pi, t)=u(x, t) .
$$

The linear equation with $\epsilon=0$ has an abundance of periodic solutions, and one can attempt to construct periodic solutions for the nonlinear case by applying the implicit function theorem around the point $u=0, \epsilon=0$. If one looks for periodic solutions with temporal period $2 \pi T$, it is convenient to represent solutions of the linear problem 


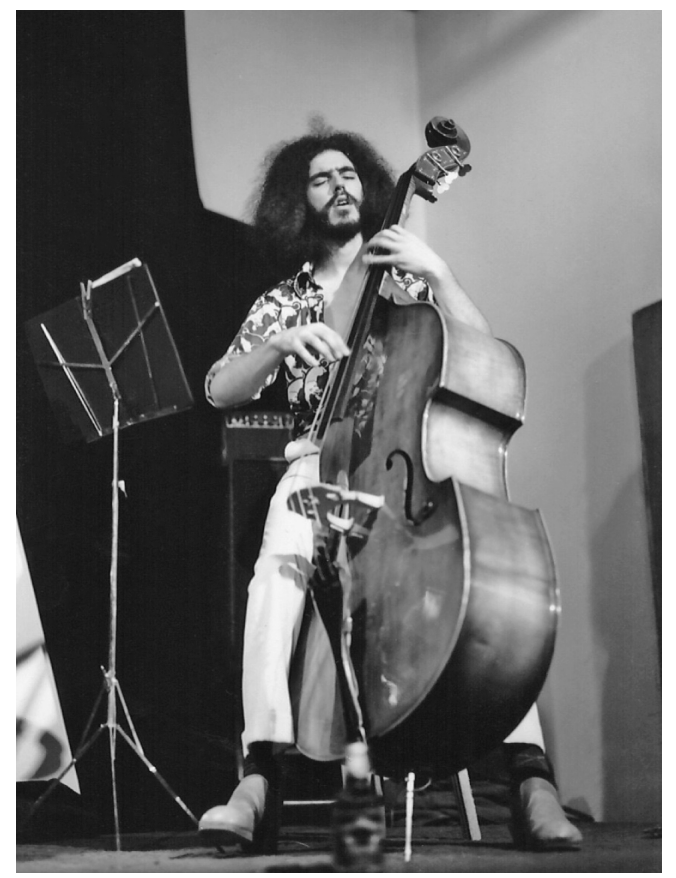

Figure 5. Walter playing his bass in Berkeley in 1973.

in terms of their Fourier expansion

$$
u(x, t)=\sum_{m, n} \hat{u}(m, n) e^{i m x} e^{i \frac{n}{T} t},
$$

and then the representation of the linearization at the zero solution is given by the linear operator

$$
(L v)(x, t)=-\sum_{m, n}\left(m^{2}-\left(\frac{n}{T}\right)^{2}\right) \hat{v}(m, n) e^{i m x} e^{i \frac{n}{T} t},
$$

and hence the inverse of the linearization is given by

$$
\left(L^{-1} v\right)(x, t)=-\sum_{m, n} \frac{\hat{v}(m, n)}{\left(m^{2}-\left(\frac{n}{T}\right)^{2}\right)} e^{i m x} e^{i \frac{n}{T} t} .
$$

Note that if $T$ is a rational number, the inverse is undefined-the values of $(m, n)$ for which the denominator vanishes are known as "resonances." However, even if $T$ is irrational, the denominator can be arbitrarily small for choices of $(\mathrm{n} / \mathrm{m})$ that give a good rational approximation to $T .{ }^{1}$ These are the "small divisors" referred to above, and as a consequence $L^{-1}$ is not a bounded operator and one cannot apply the implicit function theorem to construct periodic solutions as one does in the Lyapunov Center Theorem in the study of ordinary differential equations (ODEs).

\footnotetext{
${ }^{1}$ Because of the form of the dispersion relation of the wave equation there is a set of irrational periods $T$ for which the denominator in (7) is bounded away from zero for all pairs of integers $(m, n)$. These irrational numbers form a set of measure zero, and for more typical dispersion relations, any irrational choice of $T$ will lead to arbitrarily small divisors for some choices of $(m, n)$.
}

It was a shared interest in this question that led Walter and me to begin to collaborate. While there had been previous results on related questions using KolmogorovArnold-Moser techniques, Walter's and my approach was inspired by Nash-Moser "hard" implicit function theorems in which the solution is constructed by a Newton's method type of iterative construction. This approach is less tied to the Hamiltonian nature of the problem and in principle permits the extension of these ideas to nonHamiltonian examples. At the first stage of the iteration one encounters a linear operator like $L$ in (6), and by making assumptions about how well the irrational number $T$ can be approximated by rationals (assumptions that are satisfied for almost every irrational number $T$ ), one can control the inverse of $L$ with a prescribed loss of smoothness. At subsequent stages of the iteration, however, one must also include the linearization of the nonlinear term about the approximate solution. This leads to a linear operator that turns out to have many similarities with the Schrödinger operator with a random potential function, and by exploiting ideas that originated in the study of Anderson localization, Walter and I were able to prove that the iterative construction converged and that one had periodic solutions for (4) for all periods except for a set of arbitrarily small measure [16].

The techniques used in the construction of periodic solutions were also reminiscent of normal form transformations used in the study of ODEs. Walter used and extended the theory of normal forms in a variety of PDE contexts, most notably in the study of water waves. Starting from Zakharov's Hamiltonian formulation of the water wave problem, Walter showed (in collaboration with P. Worfolk [17]) that the fourth-order Birkhoff normal form transformation of the water wave Hamiltonian leads to a completely integrable Hamiltonian system but that there are families of nonvanishing resonant terms in the fifthorder Birkhoff normal form that make it very unlikely that the full water wave problem is integrable. In further, very influential, work with M. Groves [7] and C. Sulem and P. L. Sulem [15], Walter also exploited the Hamiltonian formulation of the water wave problem to derive and justify modulation equations like the Korteweg-de Vries and nonlinear Schrödinger equations that serve as approximations to the water wave equations in appropriate asymptotic regimes.

More recently, Walter's interests turned to a study of the normal form theory for PDEs defined on unbounded spatial domains. Here, rather than sums of the type appearing in (7), the normal form transformations are defined by integrals in which the denominator of the integrand contains linear combinations of the frequencies of the linearized problem. As in (7) these combinations of 
frequencies may vanish at resonances, but in contrast to the case considered above where a resonance means that the sum defining the inverse is undefined, an integral may have a singular kernel and still lead to a well-defined transformation. This is related to H. McKean's question, "How real is resonance?" Craig recently proved (in collaboration with Selvitella and Wang [13]) that for the nonlinear Schrödinger equation defined on $\mathbb{R}^{n}$, one can define a Birkhoff normal form transformation, on appropriate Sobolev spaces and in a neighborhood of the origin, that not only eliminates all nonresonant cubic terms in the Hamiltonian (as is typical in finite-dimensional problems) but also all resonant terms as well. Thus, at least in this case, the resonances seem less important in this infinitedimensional context than in finite dimensions. These very recent results open a host of interesting questions, such as whether or not one can iterate this procedure to eliminate higher-order terms from the Hamiltonian and whether or not one can define the Birkhoff normal form transformation on other Sobolev spaces (e.g., the energy space for the equation) which are more naturally related to the dynamics of the problem. Walter would undoubtedly have been deeply involved in the search for answers to these questions had it not been for his untimely death.

While these papers focussed on the Hamiltonian aspects of these PDEs, Walter also made significant contributions to the understanding of dispersive phenomena in nonlinear equations using techniques from microlocal analysis. In several papers with T. Kappeler and W. Strauss, culminating with [12], Walter analyzed the microlocal dispersive smoothing effect for the linear Schrödinger equation $2 i \partial_{t} \psi+\sum_{j, \ell=1}^{n} \partial_{x^{j}} a^{j \ell}(x) \partial_{x} \psi$ and established a connection between the microlocal smoothness of solutions of the initial value problem and the global behavior of bicharacteristics of the principal symbol.

My collaborations with Walter were among the highlights of my career. He brought deep insights and a broad mathematical background to bear on our joint projects, and he had very high standards for his work. In addition, his unfailing optimism and good cheer made collaborating with him a real joy. He certainly had much more to contribute to our field, but his spirit and vision will live on through the students and postdocs he trained and guided and the friends and colleagues who profited from his perspective and understanding.

\section{Walter Craig: A Mathematician and a Friend So Present in Our Community}

\section{Claude Bardos}

As it is often the case in our community of mathematicians, a few years before I met him, I already knew of Walter by reading his contribution on the derivation of the Boussinesq and Korteweg-de Vries equations. With convenient scaling and sharp harmonic analysis, in the style of his advisor, Louis Nirenberg, he gave a full mathematical derivation of these equations from fundamental principles.

This was a tour de force and a forerunner of the later career of Walter. He was dealing with a problem that was important for the community and yet unsolved. To do it, he merged the tools of harmonic analysis with an excellent intuition of the physical meaning of the solutions.

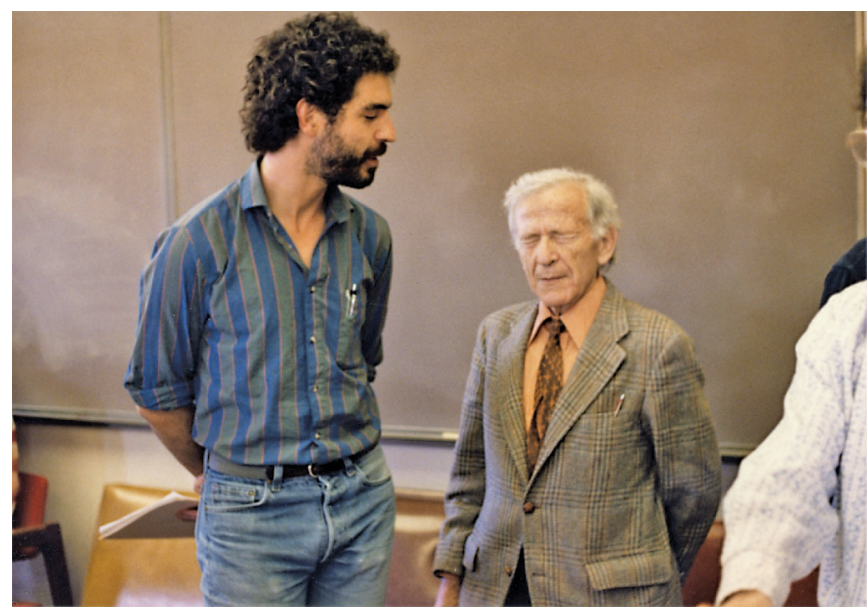

Figure 6. Walter and Hans Lewy at Stanford in 1986.

I met him in 1984 when he visited the École Normale. We were all fascinated by his charismatic behavior both in science and also in other issues of life, in particular, his availability for the warmest friendship. His fluent control of French, due to one year spent in a French high school when his father was visiting Grenoble University, made him even more appreciated by the French mathematical community. In 1988, he was one of the key speakers at a workshop organized by junior members of the French mathematical community on Oléron Island.

Claude Bardos is a professor emeritus of mathematics at University of Paris 7. His email address is $\mathrm{Cl}$ aude. bardos@gmai 1 .com. 
By that time, he had started a fruitful collaboration with Catherine Sulem. Following the program of Zakharov, they introduced a Hamiltonian formulation of the water waves problem using the Dirichlet-Neumann operator. This was the origin of many other contributions, with Pierre Louis Sulem, Philippe Guyenne, and also with experimentalists Joe Hammack and Diane Henderson, devoted to different aspects of the water wave problems.

Over the years, besides being invited to the most prestigious institutes around the world, he remained in close contact with French mathematicians, making regular short- and long-term visits in France, often with his wife Deirdre Haskell, a logician who is also a professor at McMaster University and collaborator of the Logic Group at the University of Paris 7.

I had the chance to visit him many times at Brown and later in Toronto. Being welcomed by Walter and his family, Deirdre and Zoé, was always productive from the scientific point of view and also stimulating for other aspects of life.

During his too-short life he had an important impact on the research programs of the international mathematical community. In the meantime, as a friend, his support coming with generosity and a great sense of humor was also essential for many of us. Walter will be greatly missed.

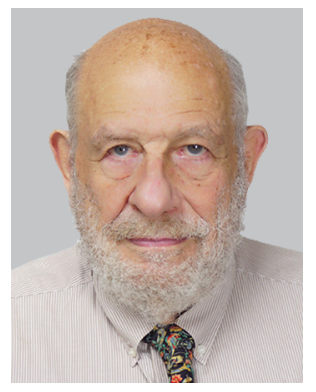

Claude Bardos

\section{Walter's Brilliant Insights into Physics}

\section{Robert Brandenberger}

Walter's premature passing is a great loss to both me and my wife Rushen. We met Walter and Deirdre during the time when both Walter and I were on the faculty of Brown University-Walter in the mathematics department and I in the physics department. Walter and Deirdre became our closest friends, always willing to open up their

Robert Brandenberger is a professor of physics at McGill University. His email address is rhb@physics.mcgi11.ca.
Providence home to us visitors commuting between Providence and Vancouver. Our close contacts continued after we all moved to Canada-Rushen and I to Montreal, Deirdre and Walter to Hamilton.

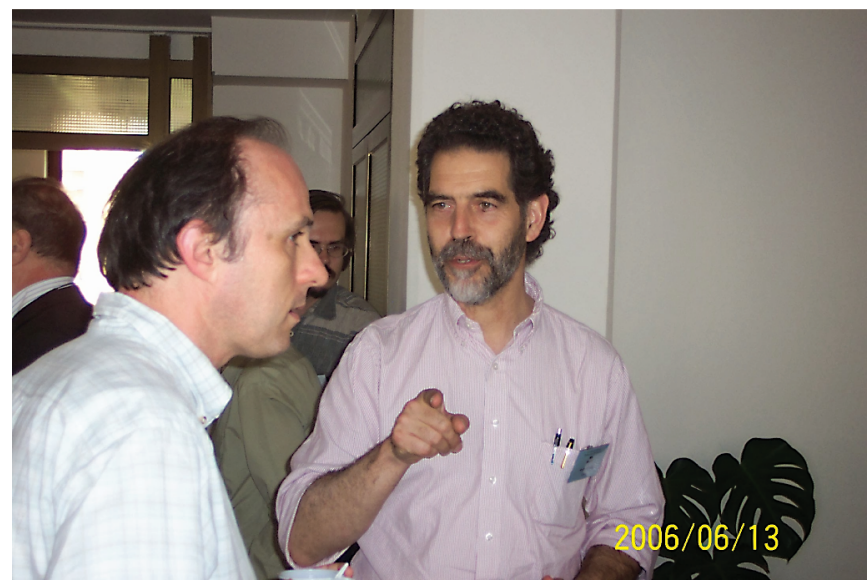

Figure 7. Walter Craig and Patrick Gerard at the Workshop on Mathematical Hydrodynamics at the Steklov Institute (2006).

As a lowly cosmologist I do not have the background to truly appreciate Walter's brilliance as a mathematician, but I learned to appreciate his deep appreciation of and insights into physics. Here is one story that demonstrates this. In 1990, fellow cosmologist Jennie Traschen (University of Massachusetts) and I had realized that the heating of the universe that takes place at the end of the hypothetical period of inflation proceeds via a parametric resonance instability. A number of years after we proposed this mechanism, it started to become very popular under the name "preheating." But I became worried about the possible effects of noise rendering the effect inefficient. Initial numerical work by a visiting scientist, however, did not show any evidence of the particular type of noise that we were considering reducing the efficiency of the instability. At some casual meeting I told Walter about this problem, and he became interested. A couple of days later he realized that the general mathematical framework of Floquet theory coupled with arguments from random matrix theory could be used to prove that the particular type of noise in fact rendered the instability stronger $[23,24]$. It is at this point that Walter's brilliant physics insight showed: he realized that the classical dynamical systems problem we were considering could be mapped onto the problem of the time-independent Schrödinger equation in a periodic potential in the presence of aperiodic noise (in one space dimension) and that our work in fact provided a new proof of Anderson localization [5]. No cosmologist I interacted with at that time had made this connection. 


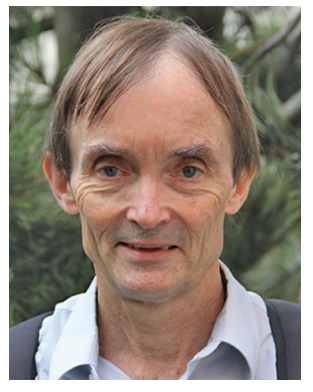

Robert

Brandenberger

\section{My Friend Walter}

\section{Sergei Kuksin}

I first visited the West in 1990, when I stayed for nine months in Bonn with my family. During this time, I met a number of colleagues who before that I knew only by their work-among them, Walter. Since then we remained friends, and I often enjoyed the opportunity to visit with Walter, his wife Deirdre, and daughter Zoé at their home in Hamilton. I got his last email January 2. It contained the best New Year wishes to all of us, and as with all his messages, remained optimistic even when his health was not so good.

Walter loved all of mathematics. He was not restricted to a few research fields-he was always looking around, making sharp and nonstandard observations. Water waves were one of his main interests, but he also made important contributions to the fundamental equations of gas and hydrodynamics. His paper [4] is dedicated to the Boltzmann equation, which is notoriously complicated in high dimensions but is well-posed in one dimension. In this work, Walter and his collaborators observed that it remains well-posed in the "mixed case," when the space variable is one-dimensional periodic while the velocity is threedimensional. Such equations describe shock-like motion of gas in a narrow tube.

Turning to viscous fluids, Walter made a number of important contributions to the three-dimensional NavierStokes equations, together with young mathematicians who were then postdoctoral fellows at McMaster. In [3], Walter and coauthors identify a subclass of weak solutions that satisfy a localized version of the energy inequality. They observe that such solutions obey the regularity property of Caffarelli-Kohn-Nirenberg and examine

Sergei Kuksin is Directeur de Recherche, CNRS, Institut de Mathématiques de Jussieu, Université Paris-Diderot. His email address is kuksin@math .jussieu.fr. which construction of the weak solutions gives rise to solutions of this subclass. In [2], Walter and collaborators obtain new estimates on the Fourier transform of weak solutions for the 3D Navier-Stokes equations and derive from them rigorous inequalities on the energy spectra of solutions that agree with the Kolmogorov-Obukhov law from the theory of turbulence. In the related work [1], Walter and M. Arnold obtain microlocal lower bounds on the singular sets of weak solutions of 3D Navier-Stokes equations, which supplement the well-known upper bounds of Caffarelli-Kohn-Nirenberg.

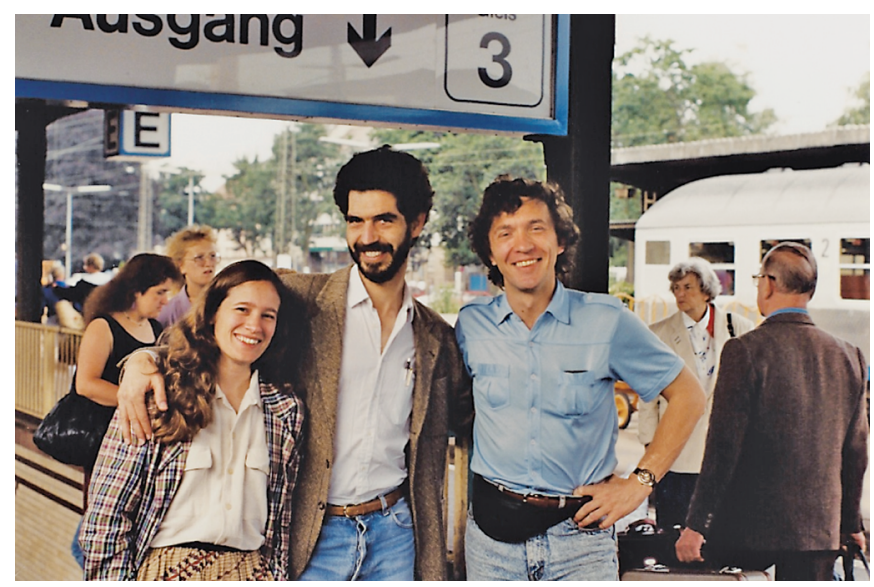

Figure 8. Deirdre Haskell, Walter Craig, and Sergei Kuksin in Germany in 1991.

One of my former PhD students, Andrei Biryuk, was a postdoc with Walter, and he reported that as a mentor, "Walter was exceptionally kind, good and friendly. He was always ready to help other people, admired mathematics and had wide erudition. His postdocs remember nostalgically infinite meetings with him in cafeterias, when considerable calculations were performed on napkins, and proofs of theorems were finalized." He said that Walter had several requirements or wishes for his postdocs, which included: the postdoc must (i) be happy, (ii) be healthy, (iii) be financially secure, (iv) work hard and enjoy mathematics. Andrei said that he was impressed by these requirements, which encapsulate Walter's concern for others, especially younger members of the mathematics community.

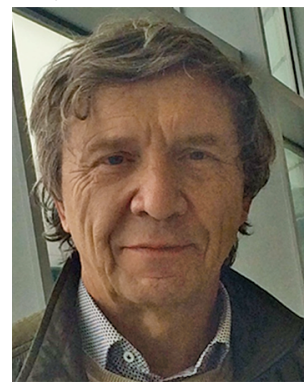

Sergei Kuksin 


\section{Recollections of Walter in France}

\section{Jean-Claude Saut}

I first met Walter in Orsay in the eighties. I remember how he explained to me the details of his beautiful and seminal result on the first rigorous derivation of the Korteweg-de Vries (KdV) equation, actually formally derived by Boussinesq in 1877 . This was a real tour de force, involving the proof of the first long-time existence for the full water wave system (derived by Lagrange in 1781!) together with the optimal error estimate of the $\mathrm{KdV}$ approximation in the Boussinesq regime.

This paper was just the first in a long series where Walter made fundamental contributions to the water waves theory. Here are some striking ones:

Following an idea of Zakharov, he gave (with Catherine Sulem) the elegant formulation of the water waves system using the Dirichlet-Neumann operator, universally used since and known as the Zakharov-Craig-Sulem formulation. This formulation leads to a very efficient numerical method for the simulation of water waves and is the starting point of all the rigorous justifications of the plethora of asymptotic models of water waves in various regimes.

Among his other contributions (some obtained in collaboration, in particular with Philippe Guyenne and Catherine and Pierre-Louis Sulem), I could mention the rigorous justification of the Davey-Stewartson system in the modulational regime and a series of very nice papers on the rigorous modeling of internal waves and of their interactions with surface waves, including the case of a nontrivial bathymetry.

While interested in the theoretical aspects of water waves, Walter did not forget the world of "real" waves, as his close collaboration with the experimentalists Joe Hammack and Diane Henderson shows.

During our first meeting I was struck by his very good French, which involved from time to time some high school slang expressions. He later told me that he spent one year in Grenoble as a high school student on the occasion of an invitation of his father to Grenoble University. He was then very successful in the math class, but he was expelled from the English class, his teacher being probably bothered that his English was better than hers.

We remained very close friends from that time and we often met abroad or in France, where he had a lot of friends and where he made frequent long-term visits (Bordeaux,

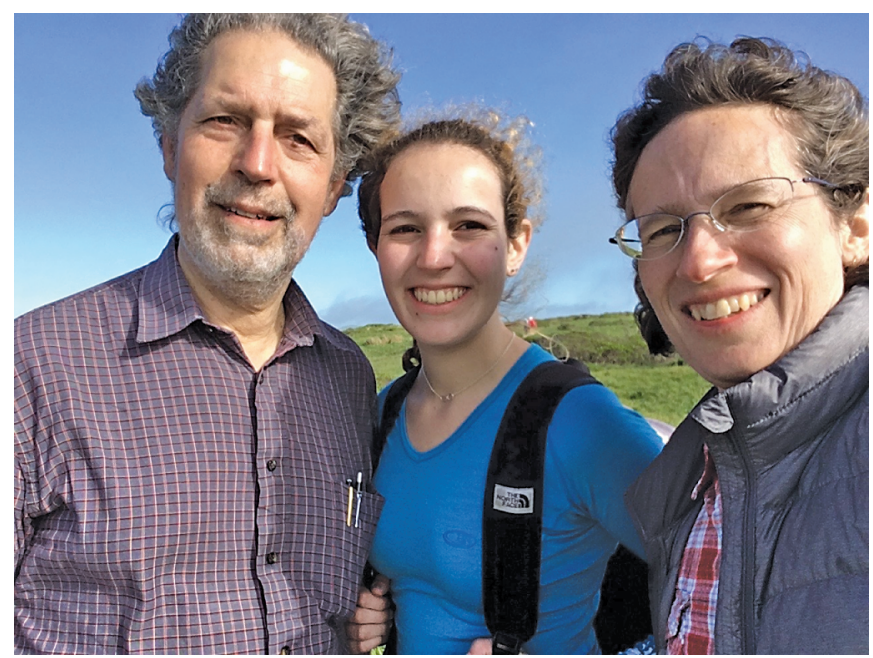

Figure 9. Walter Craig, Zoé Haskell-Craig, and Deirdre Haskell at Point Reyes, California, 2017.

Paris 6, Paris 7, Dauphine, Orsay, CMLA-ENS Cachan, Cergy, Toulouse, ENS, IHES...). On the occasion of a visiting professorship in Cachan he gave a course on small divisors, leading to a volume published in 2000 in the SMF series Panoramas et Synthèses (Problèmes de petits diviseurs dans les équations aux dérivées partielles).

Walter could at the same time master and bring fundamental contributions to the more delicate aspects of Hamiltonian systems (a recurrent joke was to point out to him that it was a sign of destiny that he happened to become professor in Hamilton) and to the modeling and numerical simulations of water waves, including interactions with oceanographers and experimentalists.

Walter was a great and brilliant scientist and a remarkable human being. We will greatly miss him.

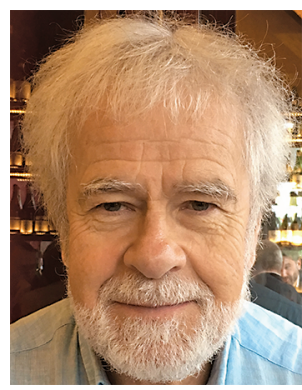

Jean-Claude Saut

Jean-Claude Saut is a professor at the Laboratoire de Mathématiques d'Orsay, Université Paris-Saclay. His email address is jean-claude.saut Quniversite-paris-saclay.fr. 


\section{References}

[1] M. Arnold and W. Craig, On the size of the NavierStokes singular set, Discrete Contin. Dyn. Syst. 28 (2010), no. 3, 1165-1178, DOI 10.3934/dcds.2010.28.1165. MR2644785

[2] A. Biryuk and W. Craig, Bounds on Kolmogorov spectra for the Navier-Stokes equations, Phys. D 241 (2012), no. 4, 426438, DOI 10.1016/j.physd.2011.10.013, MR2872819

[3] A. Biryuk, W. Craig, and S. Ibrahim, Construction of suitable weak solutions of the Navier-Stokes equations, Stochastic analysis and partial differential equations, Contemp. Math., vol. 429, Amer. Math. Soc., Providence, RI, 2007, pp. 1-18, DOI 10.1090/conm/429/08226. MR2391525

[4] A. Biryuk, W. Craig, and V. Panferov, Strong solutions of the Boltzmann equation in one spatial dimension (English, with English and French summaries), C. R. Math. Acad. Sci. Paris 342 (2006), no. 11, 843-848, DOI 10.1016/j.crma.2006.04.005. MR2224633

[5] R. Brandenberger and W. Craig, Towards a new proof of Anderson localization, Eur. Phys. J. C 72 (2012), 1881.

[6] W. Craig, An existence theory for water waves and the Boussinesq and Korteweg-de Vries scaling limits, Comm. Partial Differential Equations 10 (1985), no. 8, 787-1003, DOI 10.1080/03605308508820396 MR795808

[7] W. Craig and M. D. Groves, Hamiltonian long-wave approximations to the water-wave problem, Wave Motion 19 (1994), no. 4, 367-389, DOI 10.1016/0165-2125(94)90003-5. MR1285131

[8] W. Craig, P. Guyenne, J. Hammack, D. Henderson, and C. Sulem, Solitary water wave interactions, Phys. Fluids 18 (2006), no. 5, 057106, 25, DOI 10.1063/1.2205916 MR2259317

[9] W. Craig, P. Guyenne, and H. Kalisch, Hamiltonian longwave expansions for free surfaces and interfaces, Comm. Pure Appl. Math. 58 (2005), no. 12, 1587-1641, DOI 10.1002/cpa.20098, MR2177163

[10] W. Craig, P. Guyenne, and C. Sulem, Water waves over a random bottom, J. Fluid Mech. 640 (2009), 79-107, DOI 10.1017/S0022112009991248 MR2565938

[11] W. Craig, P. Guyenne, and C. Sulem, The surface signature of internal waves, J. Fluid Mech. 710 (2012), 277-303, DOI 10.1017/ifm.2012.364. MR2993653

[12] W. Craig, T. Kappeler, and W. Strauss, Microlocal dispersive smoothing for the Schrödinger equation, Comm. Pure Appl. Math. 48 (1995), no. 8, 769-860, DOI 10.1002/cpa.3160480802 MR1361016

[13] W. Craig, A. Selvitella, and Y. Wang, Birkhoff normal form for the nonlinear Schrödinger equation, Atti Accad. Naz. Lincei Rend. Lincei Mat. Appl. 24 (2013), no. 2, 215-228, DOI 10.4171/RLM/653. MR3073241

[14] W. Craig and C. Sulem, Numerical simulation of gravity waves, J. Comput. Phys. 108 (1993), no. 1, 73-83, DOI 10.1006/jcph.1993.1164. MR1239970
[15] W. Craig, C. Sulem, and P.-L. Sulem, Nonlinear modulation of gravity waves: a rigorous approach, Nonlinearity 5 (1992), no. 2, 497-522. MR1158383

[16] W. Craig and C. E. Wayne, Newton's method and periodic solutions of nonlinear wave equations, Comm. Pure Appl. Math. 46 (1993), no. 11, 1409-1498, DOI 10.1002/cpa.3160461102 MR1239318

[17] W. Craig and P. A. Worfolk, An integrable normal form for water waves in infinite depth, Phys. D 84 (1995), no. 3-4, 513-531, DOI 10.1016/0167-2789(95)00067-E MR1336546

[18] P. Germain, N. Masmoudi, and J. Shatah, Global solutions for the gravity water waves equation in dimension 3, Ann. of Math. (2) 175 (2012), no. 2, 691-754, DOI 10.4007 /annals.2012.175.2.6. MR2993751

[19] D. Lannes, The water waves problem: Mathematical analysis and asymptotics, Mathematical Surveys and Monographs, vol. 188, American Mathematical Society, Providence, RI, 2013. MR3060183

[20] W. Craig, P. Guyenne, D. P. Nicholls, and C. Sulem, Hamiltonian long-wave expansions for water waves over a rough bottom, Proc. R. Soc. Lond. Ser. A Math. Phys. Eng. Sci. 461 (2005), no. 2055, 839-873, DOI 10.1098/rspa.2004.1367 MR2121939

[21] L. Xu and P. Guyenne, Numerical simulation of three-dimensional nonlinear water waves, J. Comput. Phys. 228 (2009), no. 22, 8446-8466, DOI 10.1016/j.jcp.2009.08.015 MR2574099

[22] V. E. Zakharov, Stability of periodic waves of finite amplitude on the surface of a deep fluid, J. Appl. Mech. Tech. Phys. 9 (1968), 190-4.

[23] V. Zanchin, A. Maia, W. Craig, and R. Brandenberger, Reheating in the presence of noise, Phys. Rev. D 57 (1998), 4651.

[24] V. Zanchin, A. Maia, W. Craig, and R. Brandenberger, Reheating in the presence of inhomogeneous noise, Phys. Rev. D 60 (1999), 4651.

Credits

Figure 1 is by Jürgen Pöschel.

Figures 2 and 5 are courtesy of Deborah Craig.

Figures 3 and 4 are by Philippe Guyenne.

Figures 6 and 9 are used with permission of Deirdre Haskell.

Figures 7 and 8 are by C. Eugene Wayne.

Photo of Catherine Sulem is courtesy of Catherine Sulem.

Photo of C. Eugene Wayne is courtesy of C. Eugene Wayne.

Photo of Philippe Guyenne is courtesy of Philippe Guyenne.

Photo of Claude Bardos is courtesy of Claude Bardos.

Photo of Robert Brandenberger is courtesy of Christina Buchmann.

Photo of Sergei Kuksin is courtesy of Sergei Kuksin.

Photo of Jean-Claude Saut is courtesy of Jean-Claude Saut. 